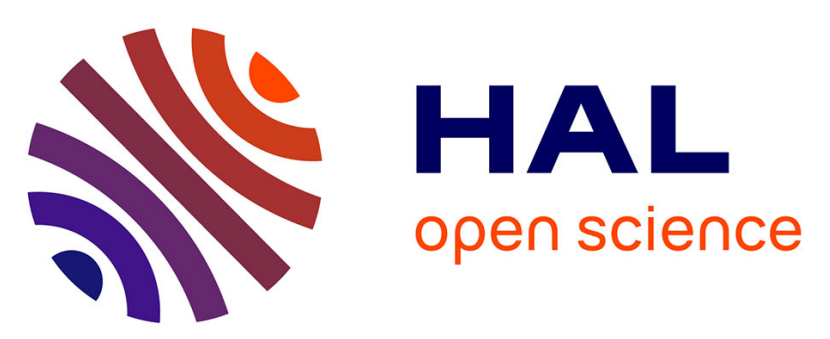

\title{
Apport des modèles murins à la physiopathologie des cardiomyopathies diabétiques
}

\author{
L. Pereira, J.-J. Mercadier
}

\section{To cite this version:}

L. Pereira, J.-J. Mercadier. Apport des modèles murins à la physiopathologie des cardiomyopathies diabétiques. Archives des Maladies du Coeur et des Vaisseaux - Pratique, 2019, 2019, pp.24 - 27. 10.1016/j.amcp.2018.11.005 . hal-03486244

\section{HAL Id: hal-03486244 \\ https://hal.science/hal-03486244}

Submitted on 20 Dec 2021

HAL is a multi-disciplinary open access archive for the deposit and dissemination of scientific research documents, whether they are published or not. The documents may come from teaching and research institutions in France or abroad, or from public or private research centers.
L'archive ouverte pluridisciplinaire HAL, est destinée au dépôt et à la diffusion de documents scientifiques de niveau recherche, publiés ou non, émanant des établissements d'enseignement et de recherche français ou étrangers, des laboratoires publics ou privés.

\section{다)(1) $(5$}

Distributed under a Creative Commons Attribution - NonCommercial| 4.0 International 


\title{
PHYSIOPATHOLOGIE CARDIOVASCULAIRE
}

\section{Apport des modèles murins à la physiopathologie des cardiomyopathies diabétiques}

\section{Pathophysiology of diabetic cardiomyopathy in mice models}

\author{
L. Pereira ${ }^{a}$, J.-J. Mercadier ${ }^{a, b}$
}

a. UMR-S 1180, Inserm, Univ. Paris-Sud, Université Paris-Saclay, 5 rue Jean-Baptiste Clément, 92296 Châtenay-Malabry, France.

b. Université Paris Diderot, Sorbonne Paris Cité, UFR de médecine, 16 rue Henri Huchard, 75018 Paris, France

laetitia.pereira@u-psud.fr

De nombreux cardiologues restent dubitatifs quant à l'existence d'une authentique cardiomyopathie diabétique (CMD). Pourtant, la première identification d'une dysfonction ventriculaire chez des patients diabétiques indemnes de tout autre facteur causal (atteinte des gros troncs coronaires, hypertension artérielle, valvulopathie, obésité, dyslipidémie...) remonte déjà à 1972 [1]. Ce diagnostic d'élimination est d'ailleurs à la base de la définition retenue par les différentes sociétés savantes [2]. La persistance d'un doute semble plutôt liée, d'une part au poids des facteurs confondants rencontrés chez la plupart des patients diabétiques présentant une dysfonction ventriculaire gauche qui rejette au second plan les possibles conséquences de la " simple » altération du contrôle glycémique et d'autre part, à une physiopathologie dont la complexité et la multiplicité des acteurs empêche d'en dégager un mécanisme simple et univoque, applicable à toutes les présentations cliniques.

L'existence de deux types de diabète, un diabète de type 1 (DT1) avec son défaut de sécrétion d'insuline et un diabète de type 2 (DT2) associant résistance à l'insuline et hyperinsulinémie plaide, malgré l'élévation chronique de la glycémie commune aux deux types, en faveur de deux physiopathologies distinctes. Si l'on rajoute le facteur ancienneté du diabète avec une longue phase d'évolution infraclinique, il faudrait probablement parler de divers types et stades de CMD dont il faudrait préciser les physiopathologies, plutôt que d'évoquer une entité homogène de mécanisme univoque. Rien qu'en ce qui concerne le DT2, le récent travail de Ernande et al [3], faisant émerger plusieurs " clusters » de patients, va clairement en ce sens. Dans ce contexte de complexité et d'hétérogénéité, les modèles expérimentaux murins ont permis de faire de grands progrès dans la compréhension de la physiopathologie des CMD [4]. Avant de résumer les principaux apports de ces modèles, il est nécessaire de rappeler rapidement les voies de signalisation cellulaire de l'insuline et le métabolisme énergétique des myocytes cardiaques.

\section{Le métabolisme énergétique cardiaque et ses altérations au cours du diabète}

Le myocyte cardiaque est un omnivore capable de produire de l'énergie (ATP) à partir de n'importe quel substrat, caractéristique appelée flexibilité métabolique. Dans une alimentation équilibrée, les lipides représentent environ $60 \%$ de l'apport et les glucides 30 $\%$. Dans les cellules, dont les myocytes cardiaques, l'entrée du glucose se fait par l'intermédiaire du transporteur du glucose 4 (GLUT4) et celle des acides gras par 
l'intermédiaire du cluster de différenciation 36 (CD36) et d'une translocase (FAT). La sécrétion postprandiale d'insuline par les cellules $\beta$ des ilots de Langerhans stimule la translocation de ces transporteurs à la membrane pour faciliter l'entrée des substrats métaboliques dans la cellule. La translocation de GLUT 4 fait suite à la stimulation de la voie PI3K-AKT, elle-même activée par la protéine IRS-1 (pour insulin receptor substrate 1) couplée au récepteur de l'insuline. L'activation de cette voie active également la NO synthase endothéliale favorisant la vasodilatation coronaire. Une autre voie activée par la fixation de l'insuline à son récepteur est celle des MAPK (pour mitogen activated kinases) qui favorise l'hypertrophie et le remodelage délétère (maladaptive remodelling) [5]. Le récepteur de l'insuline est ainsi doué d'une double potentialité, bénéfique dans les conditions normales où la stimulation de la voie PI3K-AKT prédomine, délétère dans des situations nutritionnelles et métaboliques caractéristiques du DT2. En effet, au cours de l'insulino-résistance et/ou du DT2, CD36 se trouve préférentiellement localisé à la membrane cellulaire tandis que GLUT4 est internalisé, retournant à ses localisations intracellulaires. II en découle une diminution de la capture du glucose par la cellule et un " shift » vers l'utilisation des acides gras, résultant en une accumulation cellulaire de ces derniers responsables d'une lipotoxicité dont les deux conséquences principales sont la baisse du rendement métabolique et contractile des myocytes et l'altération de la signalisation de l'insuline. Les données actuelles semblent indiquer que le primum movens de cette altération se situerait au niveau d'IRS-1 dont l'état de phosphorylation serait altéré par des modifications de l'équilibre oxydation-réduction et des facteurs nutritionnels, faisant basculer la signalisation de l'insuline de la voie PI3K-AKT vers la voie MAPK [5]. En outre, l'hyper-insulinémie stimule également l'IGF-R1 (pour insulinlike receptor 1), ce qui participe à l'hypertrophie des myocytes. Ces différentes altérations se retrouvent dans les modèles expérimentaux de DT1 et DT2 associées à une dysfonction mitochondriale et à une augmentation de la production d'espèces réactives de l'oxygène (ROS) provenant au moins en partie des mitochondries dans les modèles de DT2, ce qui reste discuté dans les modèles de DT1 [4]. Associées à un état d'hyperactivation systémique et tissulaire du SRAA, une accumulation de produits terminaux de glycation (AGE), une inflammation de bas grade et une dysfonction du système immunitaire [5, 6], elles sont globalement responsables d'une accumulation et d'un "crosslinking " du collagène interstitiel qui participe à la dysfonction diastolique.

Au-delà des altérations tissulaires et du métabolisme énergétique des myocytes, les modèles murins ont permis également d'identifier des altérations de l'excitation, du couplage excitation-contraction et de la relaxation des myocytes cardiaques participant aux dysfonctions systolique et diastoliques rencontrées à des degrés divers dans les divers types de CMD [7].

\section{Les modèles de diabète de type 1}

Les modèles de DT1 sont bien entendu basés sur l'altération de la production d'insuline par les cellules $\beta$ des ilots de Langerhans, qu'elle soit d'origine toxique (modèle à la streptozotocine, STZ) ou liée à une manipulation génétique (souris OVE26 ou Akita). Les souris STZ qui ne développent pas toujours une hypertrophie ventriculaire gauche, ont une dysfonction à la fois systolique et diastolique dont la sévérité dépend de la durée du diabète. Bien que discutée, il semble qu'il existe une diminution de la densité du courant entrant de $\mathrm{Ca}^{2+}$ (ICa-L) dans les myocytes. En revanche, il existe un consensus sur l'altération de la libération du $\mathrm{Ca}^{2+}$ contenu dans le réticulum sarcoplasmique (RS) responsable de la contraction. Elle est visualisée sous la forme de transitoires calciques $\left(\mathrm{Ca}_{\mathrm{tr}}\right)$ dont le pic est diminué et qui ne sont pas uniformes. S'y associent des vagues calciques diastoliques potentiellement responsables de troubles du rythme par l'intermédiaire de postdépolarisations retardées. La décroissance de la $\mathrm{Ca}_{\mathrm{tr}}$ est prolongée, avec pour conséquence une relaxation ralentie qui participe à la dysfonction diastolique. Au plan moléculaire, ces altérations sont associées à une diminution de l'expression des canaux par lesquels le $\mathrm{Ca}^{2+}$ 
sort du RS (récepteurs de la ryanodine ou RyR2) et de l'expression et de la fonction de l'ATPase qui repompe le $\mathrm{Ca}^{2+}$ dans le RS pendant la diastole (SERCA2a). II existe également une diminution de l'expression et de la fonction de l'échangeur $\mathrm{Na}^{+}-\mathrm{Ca}^{2+}$ (NCX) qui expulse les $\mathrm{Ca}^{2+}$ hors de la cellule chez les souris STZ, ce qui participe à l'altération de la décroissance de la $\mathrm{Ca}_{\mathrm{tr}}$ et de la relaxation. Les souris Akita ne semblent pas présenter de dysfonction systolique ce qui pourrait être en rapport avec l'absence d'altération de l'échangeur NCX [7].

\section{Les modèles de diabète de type 2}

Les deux principaux modèles de DT2 sont basés sur un déficit de la fonction de satiété assurée par la leptine. Les souris ob/ob ont un déficit de production de leptine tandis que les souris $d b / d b$ ont une altération du récepteur de la leptine au niveau de l'hypothalamus. De façon intéressante, ces souris présentent une obésité avec résistance à l'insuline, hyperinsulinémie, élévation des concentrations plasmatiques d'acides gras et de triglycérides qui précèdent le diabète. Le phénotype métabolique des deux types de souris est similaire mais les altérations de la fonction cardiaque sont plus marquées chez les souris $d b / d b$, ce qui reflète probablement un début plus précoce et une plus grande sévérité de l'hyperglycémie. Elles présentent des altérations des fonctions systolique et diastolique accompagnées ou non d'une hypertrophie ventriculaire gauche. En ce qui concerne le cycle cellulaire de $\mathrm{Ca}^{2+}$, l'amplitude de la $\mathrm{Ca}_{\mathrm{tr}}$ est fortement diminuée ainsi que la sensibilité des myofilaments au $\mathrm{Ca}^{2+}$, altérations contribuant toutes deux à la dysfonction systolique. La diminution de l'amplitude de la $\mathrm{Ca}_{\mathrm{tr}}$ est le résultat d'un ensemble d'altérations dont une diminution de la densité des courants calciques membranaires, la diminution de l'expression des RyR2, ainsi qu'une diminution de la quantité de $\mathrm{Ca}^{2+}$ présente dans le RS [8].

\section{Un effet propre de l'hyperglycémie ?}

De nombreuses études montrent que l'hyperglycémie induit une cytotoxicité notamment par l'induction de modifications intracellulaires post-traductionnelles. Parmi celles-ci, la voie de biosynthèse des hexosamines qui modifie l'activité des protéines cytosoliques et nucléaires par ajout d'un groupe O-GlcNAc sur leur résidus sérines ou thréonines. Cette nouvelle voie de régulation, appelée O-GIcNAcylation, émerge depuis peu comme un acteur clé du diabète et de l'insuffisance cardiaque [9]. Plus récemment, l'effet propre de l'hyperglycémie et de l'OGlcNAcylation a été étudié sur des myocytes cardiaque isolés exposés à des concentrations croissantes de glucose de 1 à $5 \mathrm{~g} / \mathrm{L}$. Cette exposition entraine une fuite de $\mathrm{Ca}^{2+}$ diastolique par les RyR2 participant à la génération d'arythmies cardiaques observées lors de l'injection de dobutamine et de caféine chez les rats diabétiques [10]. De plus, l'hyperglycémie et les modifications post-traductionnelles qui en résultent, telle que l'O-GlcNAcylation, sont également responsables d'une réduction de la capture du $\mathrm{Ca}^{2+}$ dans le RS par la SERCA2a conduisant à un défaut de relaxation [11].

\section{Au-delà du diabète}

Un certain nombre de travaux semblent indiquer que des altérations métaboliques initiatrices d'une cardiomyopathie s'observent avant le stade d'hyperglycémie comme indiqué ci-dessus pour les souris $o b / o b$ et $d b / d b$ [4]. Des rats Wistar nourris avec un régime "occidental » voient leur capture d'acides gras par le myocarde augmentée grâce à une surexpression membranaire des transporteurs CD36. Les souris qui présentent une surexpression spécifiquement cardiaque du récepteur nucléaire PPAR $\alpha$ qui augmente l'expression de la plupart des gènes impliqués dans la captation, le transport et l'oxydation des acides gras présentent, en l'absence de diabète, de nombreuses caractéristiques des CMD dont la diminution de l'expression de GLUT4 et de l'oxydation du glucose, l'hypertrophie cardiaque, la dysfonction ventriculaire gauche et la diminution de l'expression de SERCA2a. Enfin, des 
souris dont le récepteur de l'insuline a été spécifiquement supprimé au niveau cardiaque (souris CIRKO) présentent un phénotype métabolique et une dysfonction cardiaque très similaire à celui des souris $o b / o b$ et $d b / d b$ soulignant l'importance de l'altération de la signalisation cardiaque de l'insuline dans la CMD, la multiplicité des phénomènes pathogènes et la nécessité de mieux comprendre les effets propres de l'hyperglycémie dans cette pathologie complexe.

\section{Déclaration de liens d'intérêts :}

Les auteurs déclarent ne pas avoir de liens d'intérêts.

\section{Références}

[1] Rubler S, Dlugash J, Yuceoglu YZ, Kumral T, Branwood AW, Grishman A. New type of cardiomyopathy associated with diabetic glomerulosclerosis. Am J Cardiol 1972;30:595-602.

[2] Authors/Task Force Members, Rydén L, Grant PJ, et al. ESC Guidelines on diabetes, pre-diabetes, and cardiovascular diseases developed in collaboration with the EASD: the Task Force on diabetes, pre-diabetes, and cardiovascular diseases of the European Society of Cardiology (ESC) and developed in collaboration with the European Association for the Study of Diabetes (EASD). Eur Heart J 2013 34:303587. Erratum in: Eur Heart J 2014;35:1824.

[3] Ernande L, Audureau E, Jellis CL, et al. Clinical implications of echocardiographic phenotypes of patients with diabetes mellitus. J Am Coll Cardiol 2017;70:1704-16.

[4] Bugger H, Abel ED. Rodent models of diabetic cardiomyopathy. Dis Model Mech 2009;2:454-66.

[5] Jia G, DeMarco VG, Sowers JR. Insulin resistance and hyperinsulinaemia in diabetic cardiomyopathy. Nat Rev Endocrinol 2016;12:144-53.

[6] Jia G, Hill MA, Sowers JR. Diabetic cardiomyopathy: An update of mechanisms contributing to this clinical entity. Circ Res 2018;122:624-38.

[7] Pereira L, Ruiz-Hurtado G, Rueda A, Mercadier JJ, Benitah JP, Gómez AM. Calcium signaling in diabetic cardiomyocytes. Cell Calcium 2014;56:372-80.

[8] Pereira L, Matthes J, Schuster I, et al. Mechanisms of [Ca2+]i transient decrease in cardiomyopathy of db/db type 2 diabetic mice. Diabetes 2006;55:608-15.

[9] Wright JN, Collins HE, Wende AR, Chatham JC. O-GlcNAcylation and cardiovascular disease. Biochem Soc Trans 2017;45:545-53.

[10] Erickson JR, Pereira L, Wang L, et al. Diabetic hyperglycaemia activates CaMKII and arrhythmias by O-linked glycosylation. Nature 2013;502:372-6.

[11] Fricovsky ES, Suarez J, Ihm SH, et al. Excess protein O-GlcNAcylation and the progression of diabetic cardiomyopathy. Am J Physiol Regul Integr Comp Physiol 2012;303:R689-99. 
A

Contrôle

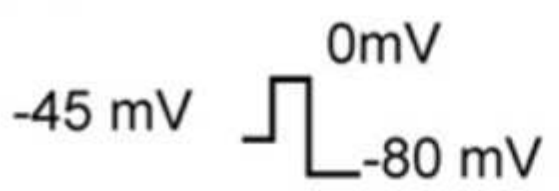

$\mathrm{db} / \mathrm{db}$
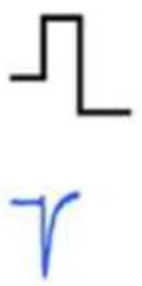

B
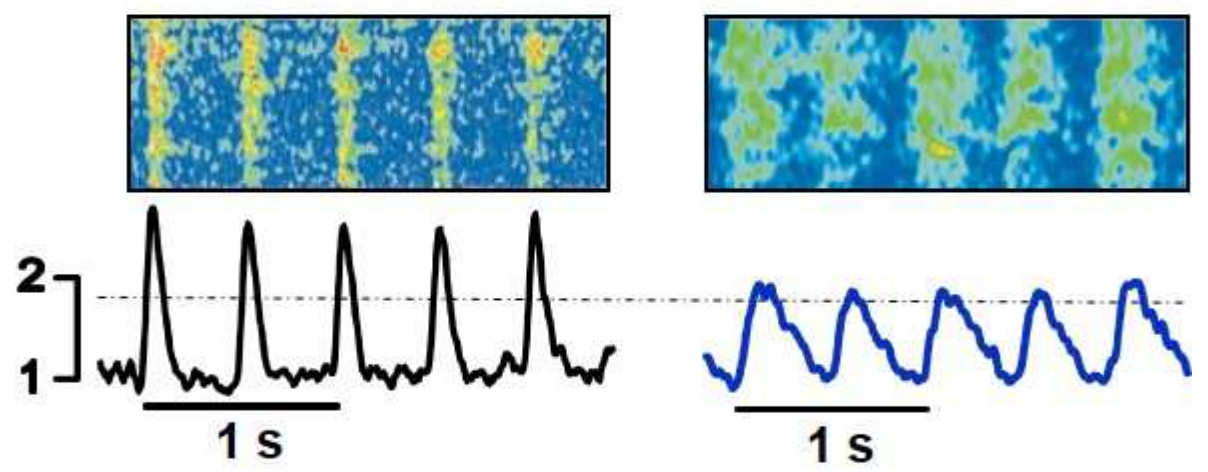

C
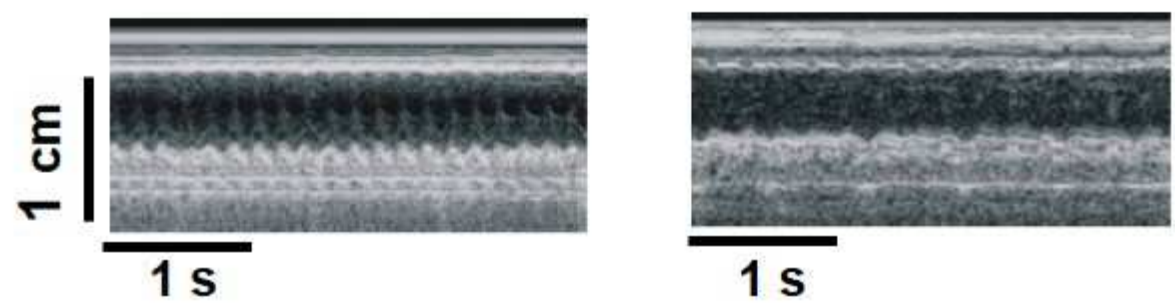

Figure. Dysfonction contractile associée aux troubles de la signalisation calcique dans un modèle de souris diabétique de type 2 (adapté de [8]).

A. Exemple de courants calciques de type $L$ enregistrés par la technique de patch-clamp sur des myocytes cardiaques ventriculaires isolées de souris contrôle et de souris diabétiques $(d b / d b)$.

B. Exemples de transitoires calciques $\left(\mathrm{Ca}_{\mathrm{tr}}\right)$ obtenues en réponse à l'influx de $\mathrm{Ca}^{2+}$ à travers les canaux calciques de type $L$ (enregistrement par couplage de la microscopie confocale avec la technique de patch-clamp).

C. Echocardiographie montrant une altération de la contraction cardiaque chez les souris $d b / d b$ comparées aux contrôles. 